\title{
RHEUMATOID ARTHRITIS
}

\section{Optimizing withdrawal of therapy to sustain remission}

Two new rheumatoid arthritis (RA) trials report sustained remission after withdrawal of methotrexate plus biologic combination therapy, one with abatacept, published in Annals of the Rheumatic Diseases, and the other with etanercept, published in The New England Journal of Medicine. Lead author of both studies, Paul Emery, says, "High remission rates were achieved with early therapy and dose reduction is feasible. These two studies are the most complete data sets available for these biologic agents."

In the phase IIIb multicentre Assessing Very Early RA Treatment (AVERT) study, eligible patients with active early RA (mean 28-joint disease activity score with C-reactive protein [DAS28-CRP] 5.4; mean symptom duration 0.56 years) were randomized to treatment with weekly doses of either methotrexate plus $125 \mathrm{mg}$ abatacept $(n=119)$ or monotherapy with abatacept $(n=116)$ or methotrexate $(n=116)$.

Consistent with other studies, remission (DAS28-CRP <2.6) at 12 months was achieved by $60.9 \%(n=70)$ of patients in the combination therapy, $42.5 \%(n=48)$ in the abatacept monotherapy and $45.2 \%$ $(n=52)$ in the methotrexate monotherapy groups. $70.6 \%$ (84/119), 56.9\% (66/116) and $62.9 \%(73 / 116)$ of patients from the three groups, respectively, had low disease activity (DAS28-CRP <3.2) and were rapidly withdrawn from drug therapy to assess sustainability of remission. 6 months after withdrawal, sustained remission was achieved by $14.8 \%$ (17/115) of patients from the combination therapy, $12.4 \%(14 / 113)$ from the abatacept monotherapy and $7.8 \%$ (9/115) from the methotrexate monotherapy groups.

In the triphasic PRIZE study, patients with active early RA (DAS28 >3.2, symptom duration $<1$ year) who had not previously been treated with methotrexate or TNF inhibitors $(n=306)$, and who responded to a 12-month open-label induction therapy with combination methotrexate plus $50 \mathrm{mg}$ etanercept (DAS28 $<3.2$ at week 39 and $<2.6$ at week 52 ) were eligible for the second double-blind phase $(n=193)$ in which they were randomized to one of three treatment regimens: $25 \mathrm{mg}$ etanercept plus methotrexate, methotrexate monotherapy or placebo. Patients with low disease activity (DAS28 $\leq 3.2$ ) at the end of this 39-week phase were eligible for the treatment-withdrawal phase of the study $(n=131)$, which lasted a further 26 weeks.

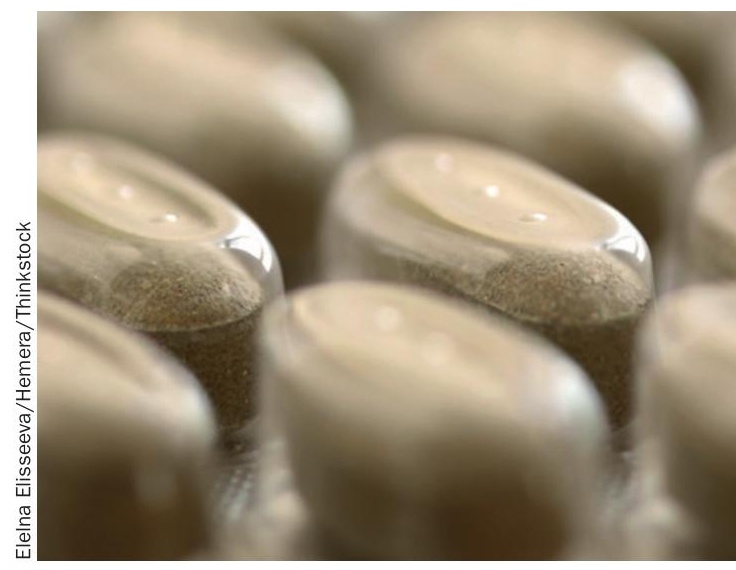

By the end of the treatment-free phase, remission (DAS28 <2.6) was maintained by more combination-treated $(n=28$, $44 \%)$ than methotrexate monotherapy $(n=19,29 \%)$ or placebo-treated patients $(n=15,23 \%)$.

Regarding future trials, Emery suggests, "We should probably aim to treat even earlier as short disease duration predicted a good long-term outcome."

Nicholas J. Bernard

Original articles Emery, P. et al. Evaluating drug-free remission with abatacept in early rheumatoid arthritis: results from the phase $3 b$, multicentre, randomised, active-controlled AVERT study of 24 months, with a 12-month, doubleblind treatment period. Ann. Rheum. Dis. doi:10.1136/ annrheumdis-2014-206106 | Emery, P. et al. Sustained remission with etanercept tapering in early rheumatoid arthritis. N. Engl. J. Med. 371, 1781-1792 (2014) 